\section{Localization of visceral pain and other sensation: before and after anesthesia}

\author{
GEORG von BÉKÉSY \\ Laboratory of Sensory Sciences \\ University of Hawaii, Honolulu, Hawaii 96822
}

The localization of sensations in sense organs that developed ontogenetically from the ectoderm layer, like vision, hearing, and skin, is much more precise than sensations inside the body, like pain in the chest or viscera, which are produced by receptors developed from the entoderm layer. Pain on the surface of the skin is much more sharply localized than is pain in the viscera. Personal experiences of pain sensations produced by an obstruction in the colon and the effects of anesthesia are described. It became evident that anesthesia can influence the activity of the vestibular organ for many days.

\section{Localization of Sensations}

It is generally accepted that pain in the chest and abdomen is poorly localized and seems to have little connection with the place of the stimulus. It can happen that pain in the abdomen is localized on the wrong side. Nevertheless, this localization can be consistent.

There is a large literature on visceral pain and pain in the chest, and there are several theories to explain them (Franz \& Iggo, 1968; Keele, 1957; Livingston et al, 1958; Livingston, 1943; Melzack \& Wall. 1965; Mendell \& Wall, 1964; Wolfson. 1966; Zimmermann, 1968). An excellent review of the subject can be found in the article by Theodore C. Ruch (1968) in Textbook of Physiology. From the point of view of practical medicine, the localization of internal pain is of utmost importance. But, unfortunately, there is no way to do scientific research on visceral pain if the pain cannot be localized properly. From the purely theoretical point of view, it is of interest why visceral pain cannot be localized, whereas sensations produced by sound and light and even taste can be

localized and correlated well with the place of the stimulus.

For instance, a sound source can be localized with a surprising accuracy in space, especially if the sound consists of short clicks. Naturally, if the sound source is in a room with reflecting walls, the stimulus is not so well defined and the localization becomes less precise. But, outdoors or in a room with damped walls, no mistakes are made.

Even the complicated sound of a violin can be attributed to an area in space with an apparent volume of the sound source and a location. To illustrate this, a violin player was asked to produce. with the strings $\mathrm{E}, \mathrm{A}, \mathrm{D}, \mathrm{G}$, sounds with equal loudness, and to describe the apparent place of the sound. Figure 1 shows such an experiment. As the pitch on the violin changes, the location of the sound source changes, too. The shift of the apparent place of the sound source is highly reproducible for the same player. It is different for different violins. The one used in Fig. 1 was a cheap one. Figure 1 shows the representative findings of one $O$. They

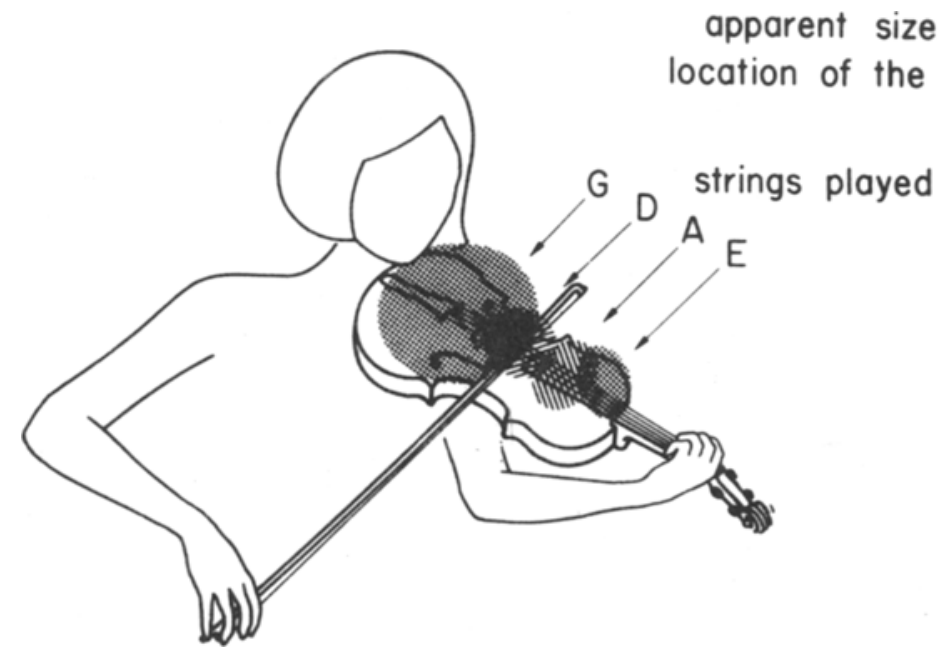

were retested for several weeks with very little changes. If the loudness of the produced sound was kept constant, the center of the sound image moved around no more than 3 to $4 \mathrm{~cm}$. The findings were reported in drawings made by the 0 .

To illustrate further how persistent localization of sound sensations are, we place on each ear an earphone, as shown in Fig. 2, and supply the two ears with the same sinusoidal tone and a loudness adjusted to be equal for both ears. Low frequencies like $500 \mathrm{cps}$ are localized in the middle of the head. But, if the frequency is increased, then the sound seems to move vertically upward in the center line and, for frequencies above $2,000 \mathrm{cps}$, it is localized outside the head. This experiment was repeated on more than $10 \mathrm{Os}$ with the same result. However, it is important to keep the loudness of the sound at speech level since for high loudness, the localization becomes less precise. Figure 2 represents the mean value of drawings made by the Os.

As is known, singers train themselves to increase the pitch of their voices higher and higher. Low frequencies, when sung, are localized again in the middle of the head They are a combination of hearing the vocal-cord vibrations by bone conduction and the sound radiated from the mouth to the ear. But, if a singer succeeds in increasing the pitch, the localization of his singing voice will move up along the middle axis of the head just as for the pure tone. This sensation is so well defined that it can be used by a singer to determine if his singing abilities are improving during the training or not. The results obtained in this simple way are just as good as when, with an acoustic frequency analyzer, the intensity of the spectrum of the singing is recorded at different frequencies.

\section{Difference between Pain and Other Sensations}

One of the most surprising facts in hearing is that the ear can handle a pressure range of $1: 10^{7}$ without introducing a distortion in a tone with a frequency of $1,000 \mathrm{cps}$. To be able to cover such a tremendous range in stimulus magnitude, it is necessary to have a large number of nerve fibers. There are in the cochlea about 30.000 hair cells, each connected with many nerve fibers. With such a large nerve

Fig. 1. Place and apparent magnitude of a sound source can be very precisely determined. This drawing shows, for a cheap violin, where the sounds produced by the four strings are localized by the player, if they are played with about the same loudness. 


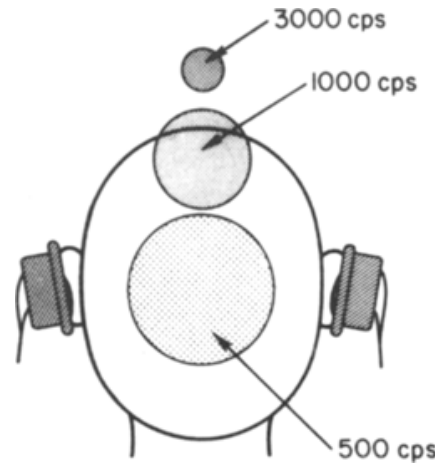

both earphones produce sounds of the same frequency and the same loudness

Fig. 2. If two equal tones are presented to both ears, then for low frequencies they are localized in the middle of the head. But, if the frequency increases, they are localized above the head. This is used by singers to find out how high the pitch of the voice is during singing.

supply, all the little details in spatial stimulus distribution can be easily recognized as such. Vibratory sensation can handle an amplitude range of 1:3,000. Therefore, we would conclude that vibrations are not so precisely localized along the surface of the skin. But for pain, the effective stimulus range is only of the order of $1: 10$. This is for pain produced by heat on the surface of the skin of the fingertip. The range is much less on the arm. We either do not feel pain or we cannot tolerate it. As a consequence of this, in a stimulus pattern produced by heat, it is difficult to recognize small variations along the surface of the skin, since there are only a few different sensation magnitude steps that can be recognized as such. A heat stimulation pattern along the surface of the skin is easily produced by winding an iron wire along a core of about $7 \mathrm{~mm}$. After the core is taken out, we obtain a spiral that can be held about $5 \mathrm{~mm}$ above the skin by an asbestos sheet. Heat radiation is produced by an electric current, and a specific heat pattern is obtained by changing the distance between the windings of the spiral along the surface.

Difference Between the Stimulation of the Ectoderm and the Entoderm Parts of the Body

Sense organs, like the ear, the eye, the skin, etc., develop from the outer laycr of the embryo, the ectoderm. Since a large part of the nervous system develops also from this layer, these sense organs have a good nerve supply. Localization of pain on the surface of the skin is quite good. This holds for pain produced by heat, mechanical, or electrical stimulation. On the other hand, the poor localization of pain in the viscera might be the consequence of the viscera developing from the entoderm layer, which has a poor nerve supply and is probably of a different type than that of the ectoderm layers. Therefore, it is not surprising that pain localization inside the body is less precise than it is outside the body.

This difference can be shown on the transition between entoderm and ectoderm layers if we stimulate the outer skin of the lip and then the inside of the mouth with a needle. As illustrated in Fig. 3, the sharpness of the localization is greater on the outer skin than it is inside the mouth, and it gets even worse if we go deeper into the throat. For these observations, the pressure of the needle on the surface was adjusted so that the sensation magnitude stayed about the same.

It is not easy to stimulate parts inside the body with vibrations. One method we used is illustrated in Fig. 4, where an electrodynamic vibrator was pressed against the lower part of the spine. Certainly these vibrations are transmitted also to the surface of the skin, but since the vibrations of the spine are the strongest, they are the only ones felt. It is an interesting experience to feel the spine vibrate. It is easily distinguished from all other sensations, is precisely located, and is felt as a vibrating rod inside the body. The vibrations are the strongest at the place where the vibrator is placed and they taper off approaching the head. The sensations of these vibrations can be well reproduced. Three Os reported about the same pattern, and any change by bending the body is easily recognized. But, still, the localization

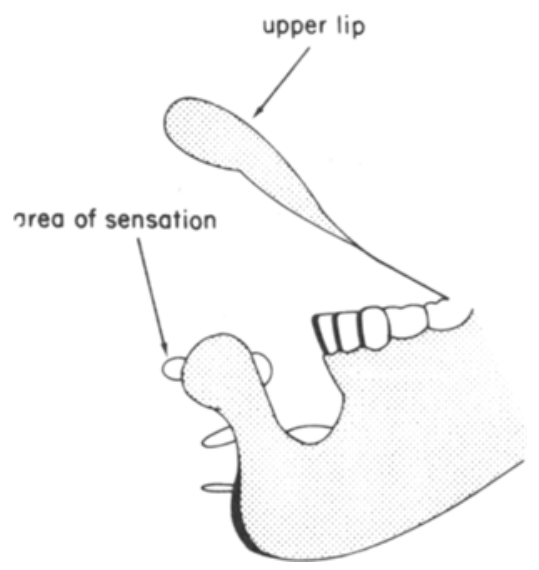

Fig. 3. Pressing the tip of a needle against the outer skin on the lip, the localization is sharp. The same tip will produce a dull and laterally spread sensation inside the mouth.

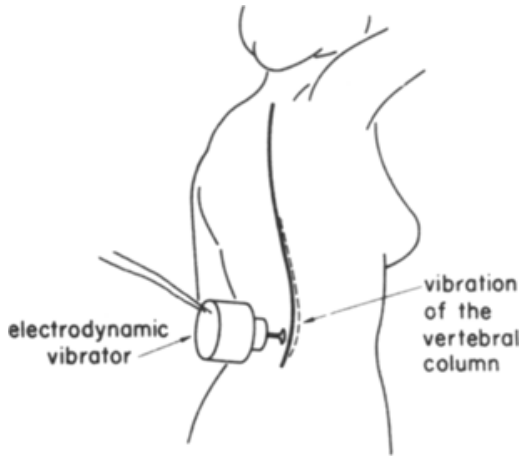

Fig. 4. A vibrator placed on the lower end of the spine will vibrate the spine so that we feel it as an isolated vibrating rod.

along the spine is not as sharp as it is outside, on the skin. The frequency used was between 30 and $50 \mathrm{cps}$, but the optimal frequency depends on the impedance of the electrodynamic driving unit and the transmission of the vibrations from the tip of the vibrator to the spine. To produce the vibrations, an electrodynamic power vibrator was used. Its maximal input was $40 \mathrm{~W}$ and it produced a peak force of $5 \mathrm{~kg}$. The natural frequency of the vibrator was about $20 \mathrm{cps}$. The tip of the vibrator was flat and had a diameter of $6 \mathrm{~mm}$ and was pressed against the spine with a force of 1 to $2 \mathrm{~kg}$. For the observations, the current through the driving system was slowly and continuously increased to an optimal value. For most of the observations, the exposure time was about $15 \mathrm{sec}$. The amplitude of the tip was not measured, since it is more a characteristic of the transmission system between the tip and the spine than it is a critical dimension of the phenomenon. Prolonged vibrations of the spine may produce headaches that may last a day.

By increasing heat stimulus on the skin above the pain threshold, pain rises very quickly, and the maximum pain is obtained shortly before the destruction of the skin. The author's personal experience is that visceral pain, produced by an obstruction in the colon, rises even faster than pain on the surface of the skin. But local visceral pain has a certain maximum that does not increase further, but instead, spreads out laterally. It is this lateral spread that makes it so difficult to localize pain correctly. 1 found that the edge of the painful area (about $18 \mathrm{~cm}$ wide, $7 \mathrm{~cm}$ high, and $4 \mathrm{~cm}$ deep) produced by an obstruction is well defined and it stays in the same place for several days. Without question, internal pain is influenced by many phenomena like central summation, inhibition, etc. It is well known that a foreign stimulus on the skin can reduce the pain sensation. But 1 


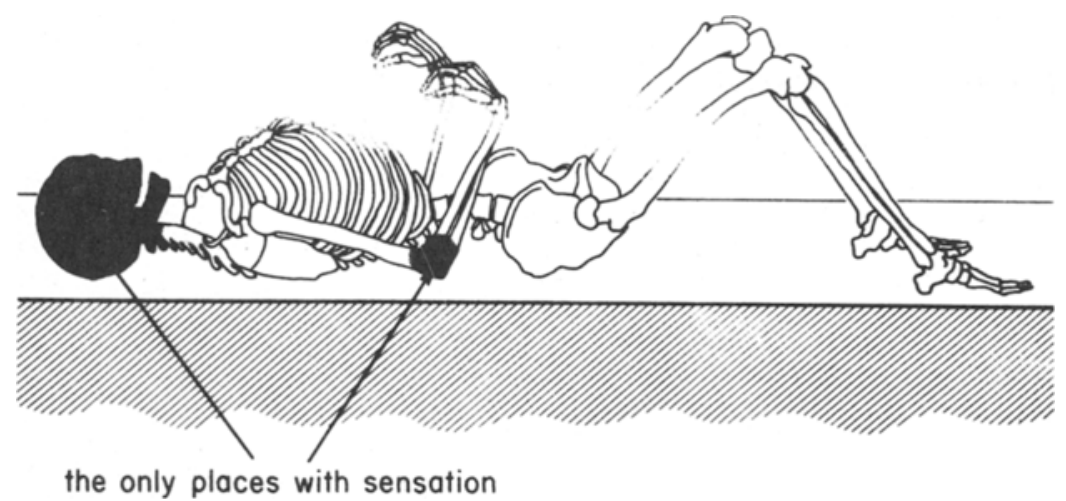

found that this is correct only for small or medium pain and not for maximal pain.

The Influence of Oxygen Intake on Sensitivity in Vision and Hearing

In experiments investigating the influence of oxygen intake on the sense organs carried out by decompression in a high-altitude chamber (simulating an altitude of about 6,000 to $8,000 \mathrm{~m}$ ), I repeatedly found that, just before losing consciousness, a piece of white paper became pinkish. A small hearing loss for the highest frequencies $(25,000 \mathrm{cps})$ was also observed. But, after regaining consciousness, there were no aftereffects influencing the sense organs. These experiments were repeated with three Os during several months with the same results. An oxygen mask was worn until the decompression reached a certain value. Then the mask was taken off and the observations were made until an assistant with an oxygen mask put the mask beck on again. Later on, sophisticated equipment was used in which the oxygen content of a nitrogen-oxygen mixture was reduced, which made it possible to stay longer at the point just before unconsciousness occurred.

From these experiments, it was concluded that a decrease of oxygen intake for a short period of time does not produce a large change in the effectiveness of the sense organs. Therefore, a possible lack of oxygen intake cannot be made responsible for phenomena observed after anesthesia.

\section{Localization of Stimuli after Anesthesia}

A short time ago, the author had to undergo a colostomy. Working in sensory perception for many decades, the question arose as to what influence the usual anesthesia during surgery has on the sense organs. The following descriptions are his own findings. However, since the surgery had to be revised after a week, there was a good opportunity to repeat all the observations made after the first surgery. The experiences described in this paper were in all details exactly the same in both cases.

I was greatly surprised to find that anesthesia has very different effects from those of diminished oxygen intake on the sense organs. The anesthetic used was Fluothane supplemented with nitrous oxide, and the depth of the anesthesia was adjusted to the needs during the surgery, which lasted about $1 \mathrm{~h}$. No preanesthetic medication was used.

The sensations I had after anesthesia are maybe best described by Fig. 5 . It seemed that the whole body was missing except the head and the two elbows, which are shaded in the figure. In both areas, a slight pain was present and, in the following hours of recovery, I became more and more aware of the vertebrae of the neck. But, even the next day, I could not localize properly the position of the hip relative to the head. Sudden pressure on the surface of the skin was still effective, but with a loss of sensitivity.

The recovery was otherwise uneventful. For example, there was no postoperative cardiovascular shock. During surgery, no arteriosclerosis of the coronary arteries could be seen. There was a change in taste quality similar to that obtained by simple dehydration, but at the same time, vision and hearing were little influenced by anesthesia. During the first few minutes, there was a red blotch in the visual field of one eye, but that disappeared very quickly.

Immediately after regaining consciousness, no disturbance in visual depth perception or in directional hearing of sounds could be observed. Echo effects in the room were suppressed to the same degree as normal, so that the room did not seem to be reverberating as it does when one listens to a speech on a tape recorder. This indicates that, on a cortical level, the recovery was in general fast, except for the processing of conflicting information. A useful method to test this is the rivalry between the visual fields of both eyes. If one is trained by stereoscopic observation to look with both eyes straight forward, a
Fig. 5. Immediately after anesthesia, it may happen that all the sensations are inhibited in a large part of the body except in the shaded areas, the head and the elbows.

simple black paper between the eyes permits one to see with one eye a different object than with the other. A fusion of the pictures of both eyes eliminates partially or totally the picture on one side. Under normal conditions, in $1.4 \mathrm{sec}$ the fused pictures change periodically from one to the other. After anesthesia, the switching time increased for me to 20 or more seconds. No partial rivalry, which is so common under normal conditions, was observed.

In spite of the small changes of these sense organs, it was obvious that the vestibular organ lost its sensitivity to a large extent. Having done in earlier years many experiments on the vestibular organ, using unilateral or bilateral caloric stimulation, I was familiar with changes in sensitivity of the vestibular organ produced by overstimulation. Especially, there was observed many times the loss of sensitivity of the horizontal canal after the Bárány test with the rotating chair. Therefore, it was easy to realize that the vestibular organ lost its sensitivity after anesthesia. I could only recognize a tilting of the head forward. The rotation of the head around its vertical axis produced no sensations at all. Why one vestibular channel is less influenced by anesthesia than the other parts is not clear. But, in a vertical position, it was easy to observe, even after 2 days, that tilting forward and backward was much better under control than any rotation of the body.

The vestibular system is not a simple input-output system. Since under normal conditions a rotation of the head stimulates the end organs on both sides in an opposite way, the sensation of rotation is evaluated from the difference between the reactions of the two sets of end organs. Maybe this is one of the reasons why anesthesia has a larger influence on the vestibular system than on vision or hearing. Besides this, the final evaluation of a head movement is influenced also by sensations in the skin, muscle, and joints. It is a complicated interaction so that they do not produce a specific sensation; otherwise no clear judgment could be obtained.

One of the most surprising experiences during the recovery period was the close parallel between the recovery of the vestibular organ and the improvement of sensations on the skin. muscles, and joints. The vestibular recovery was observed by rotations of the head around the vertical 
axis. It was not difficult to follow from day to day how, beginning from the head, the sensitivity of the vertebrae improved until control of the hip was possible.

Having experienced this type of inhibition of body sensations, it was possible to recognize that a small amount of a similar inhibition could be observed also after the routine Barany test with the rotating chair, especially so when it was repeated several times. In this test, a mechanical overstimulation of the cupula of the horizontal canal is produced. It probably takes a certain time for the cupula to swing back into its normal position.

Whether the inhibitory phenomena after anesthesia are triggered mechanically in the end organ, as in the test of the rotating chair, or centrally, by a direct effect of the anesthetic, could have been decided by a caloric test immediately after surgery.

The Importance of the Vestibular Organ and Neck Innervation for Sensations Outside the Head

As Kornhuber (1969) pointed out, the fish has no neck and, therefore, the vestibular organ gives the head and body position at the same time. But in other vertebrates this is not so, and, besides the head position, we have to determine the relative position between head and the body from stimuli produced in the neck. For this reason, the neck innervation and its connections to a vestibular organ become very important. Jongkees (1969), in his review of the vestibular organ, described research done by Barré (1902), Lieon (1928), and by Fredrickson, Schwarz, and Kornhuber (1965) to prove the importance of the neck innervation. The experiments of Barré were repeated successfully by de Jong (1967).

\section{Conclusion}

I have often wondered what happens in the nervous sytem of a cat or a monkey during electrophysiological observations. Maybe Nembutal and Dial are different in their action from Fluothane supported by nitrous oxide. But I think Fluothane is a more sophisticated anesthetic than the simple Nembutal and Dial injection given to the animals. Since the time of anesthesia is in general longer than described in this case, the effects, if there are some, should also be strong, especially since the observations are done under anesthesia and not after anesthesia. The observation that the electrophysiological records change during anesthesia indicate that there are some aspects of the nervous system that are affected by anesthesia. But transducer effects like the microphonics in hearing do not seem to be influenced.

The experiences with the neck innervation brings up the question of whether in some cases it is not justified to destroy the neck innervation and the vestibular organ, since they may have an inhibitory effect on sections of the body below the neck. The other possibility is a fascinating one, namely, to evaluate anesthetics by taking into consideration their effects on the vestibular organ and the nervous system of the neck. I think with modern chemistry it would not be difficult to find compounds that decrease the influence of the vestibular organ and thus maybe reduce recovery time.

\section{REFERENCES}

BARRÉ, J. A. Review of Neurology, 1902, I, 1246. [after Jongkees (1969)].

de JONG, J. M. B. v. Akad. Dissertation, Amsterdam, 1967. [after Jongkees (1969)].
FRANZ, D. N., \& IGGO, A. Dorsal root potentials and ventral root reflexes evoked by nonmyelinated fibers. Science, 1968, 162, 1140-1142.

FREDRICKSON, J. M., SCHWARZ, D., \& KORNHUBER, H. H. Convergence and interaction of vestibular and deep somatic afferents upon neurons in the vestibular nuclei of the cat. Acta Otolaryngologica, 1965, 61, 168.

JONGKEES, L. B. W. Physiologie und pathophysiologie des Vestibular-organes. Archiv fur klinische und experimentelle Ohren, Nasen und Kchlkopf Heilkunde, 1969, 194, 1-110.

KEELE, K. D. Anatomies of pain. Oxford: Blackwell Scientific Publications, 1957.

KORNHUBER, H. H. Physiologie und klinik des vestibularen systems. Archiv fur klinische und experimentelle Ohren, Nasen und Kehlkopf Heilkunde, 1969, 194, 111-148.

LILOU, Y. C. Akad. Dissertation. Strassburg, 1928. [after Jongkees (1969)].

LIVINGSTON, W. K., MELZACK, R., \& STOTLER, W. A. Effects of discre te brainstem lesions in cats on perception of noxious stimuiation. Journal of Neurophysiology, $1958,21,352-367$.

LIVINGSTON, W. K. Pain mechanisms. New York: MacMillan, 1943.

MELZACK, R.. \& WALL, P. D. Pain mechanisms: A new theory. Science, 1965, 150, 971-979.

MENDEL.L, L. M., \& WALL, P. D. Presynaptic hyperpolarization: A role for fine afferent fibers. Journal of Physiology (London), 1964, 172, 274-295.

RUCH, T. C. Pathophysiology of pain. In J. F. Fulton (Ed.), Textbook of physiology. Philadelphia: Saunders, 1968. Pp. 345-363.

WOLFSON, R. J. The vestibular sy'stem and its research. Philadelphia: University of Pennsylvania Press, 1966

ZIMMLRMAN, M. Dorsal root potentials after C.fiber stimulation. Science, 1968, 160. 896-898.

(Accepted for publication April 23. 1970.) 